\title{
SSVEP Recognition using Wavelet Canonical Correlation Analysis for Brain Computer Interface
}

\author{
Shaobo Liu ${ }^{\mathrm{a}}$, Fuchun Sun ${ }^{\mathrm{b}}$, Wechang Zhang ${ }^{\mathrm{c}}$ and Chuanqi Tan ${ }^{\mathrm{d}}$ \\ Department of Computer Science and Technology, Tsinghua University, State Key Lab. of \\ Intelligent Technology and Systems, Tsinghua National Laboratory for Information Science and \\ Technology (TNList) \\ athusunfc@163.com, bfcsun@tsinghua.edu.cn, 9wzszwc@163.com, dchuanqi.tan@gmail.com
}

\begin{abstract}
Keywords: Frequency Recognition, Canonical Correlation Analysis (CCA), Electroencephalogram (EEG), Steady-State Visual Evoked Potential (SSVEP), Wavelet Packet Decomposition (WPD), Brain-Computer Interface (BCl)

Abstract. Canonical correlation analysis (CCA)-based methods have been widely applied to frequency recognition in steady-state visual evoked potential (SSVEP)-based brain-computer interface (BCI). However, it is hardly to obtain optimal recognition accuracy from electroencephalogram (EEG) signal mixed with variety of noises. In this paper, we use wavelet packet decomposition to reject components which are not related to stimulus frequency in SSVEP signals to improve SSVEP recognition accuracy. Experimental results show its superior performance over other competing methods.
\end{abstract}

\section{Introduction}

A brain-computer interface $(\mathrm{BCI})$ aims at providing a direct communication pathway between human brain and external devices [1, 2, 3]. Hence, BCIs can transmit control commands and feed-backs from brain cortex without relying on normal neural pathways. In recent years, electroencephalogram (EEG) based BCIs usually use three types brain activity signals, i.e., event-related potential (ERP), steady-state visual evoked potential (SSVEP) and event-related (de)synchronization (ERD/ERS). SSVEP-based BCI has attracted increasing attention, since it can provides higher information transfer rate (ITR) with less training to the user [4, 8].

The SSVEP is periodic response potentials in the parietal and occipital areas induced by rapidly repetitive visual stimulation with a frequency higher than $6 \mathrm{~Hz}[5,6]$. It consists of a range of discrete frequency components, namely, the fundamental of the visual stimulus as well as its harmonics. Based on the truth, the key point of SSVEP-based BCIs is to recognize the SSVEP frequency components in EEG signals. Since original SSVEP signals are usually contaminated by spontaneous EEG and other noises, it is an important problem to develop an effective approach to recognize the SSVEP frequency with a high accuracy, especially at a short time window length (TW).

Traditional power spectral density based analysis (PSDA) [10] estimates the PSD values of EEG signals at different frequencies within a specific TW through Fast Fourier Transform (FFT). The frequency with maximal PSD value is taken as stimulus frequency. However, PSDA with a signal or bipolar channel is sensitive to noises and usually needs relatively long TW to estimate the spectrum with sufficient frequency resolution. These drawbacks seriously restrict its application in real-time BCI system. To improve SSVEP recognition performance, several methods have been proposed. Canonical correlation analysis (CCA)-based method, firstly introduced by Lin et al. [5], has attracted increasing attention. They used CCA to maximize correlations between the EEG signals from multiple channels and the pre-constructed sine-cosine reference signals at each of the used stimulus frequencies. The stimulus frequency with the maximal correlation coefficient was taken as the SSVEP frequency. CCA has presented superior performance in comparison with PSDA. Recently, several CCA-based methods have been proposed to further improve SSVEP recognition accuracy.

Multiway canonical correlation analysis (MCCA), proposed by Zhang et al. [7], was used to obtain the optimal reference signals from a number of trials for each specific subject. The results has shown its better performance than the standard CCA. To further improve SSVEP recognition performance, 
Zhang at al. [8] propose an L1-regularized MCCA to learn sparse projection vector with function of effective trial selection during the reference signal optimization. Although the aforementioned approaches show better performance, they pay no attention to reject frequency components which are not related to stimulus frequency in SSVEP signals. For this reason, we propose a Wavelet CCA approach to reject those useless frequency components and detect the target stimulus frequency. We compared our method with other CCA-based methods on the EEG signals from 3 healthy subjects.

\section{Proposed Approach}

SSVEP Recognition based on CCA. Canonical Correlation analysis (CCA), firstly proposed by Hotelling [9], is a multivariable statistical method, which aims at exploring the underlying correlation between two sets of data. Given two multidimensional random variables $X \in R^{I_{1} \times J}$ and $Y \in R^{I_{2} \times J}$, CCA seeks a pair of linear transform $w \in R^{I_{1}}$ and $v \in R^{I_{2}}$ to maximize the correlation between linear combinations $w^{T} X$ and $v^{T} Y$, through solving the following optimization problem:

$$
\rho=\max _{w, v} \frac{w^{T} X Y^{T} v}{\sqrt{w^{T} X X^{T} w v^{T} Y Y^{T} v}}
$$

The maximum $\rho$ denotes the maximum canonical correlation coefficient. The optimization problem in (1) can be solved by a generalized eigenvalue problem.

Lin et al. [5] firstly introduced CCA to recognize SSVEP frequency in BCI application. Assume our goal is to recognize target frequency from $M$ stimulus frequencies in an SSVEP-based BCI. $\widehat{X}=R^{C \times P}$ is an EEG signal from $C$ channels with $\underline{P}$ time points per channel. $Y_{m} \in R^{2 H \times P}$ is the reference signal at the $m$-th stimulus frequency $f_{m}(m=1,2, \cdots, M)$, which is constructed by a series of sine-cosine waves. Solving the canonical correlations $\rho_{m}$ between EEG data $\widehat{X}$ and reference signal $Y_{m}(m=1,2, \cdots, M)$ by (1), the SSVEP target frequency is taken as

$$
f_{t}=\max _{f_{m}} \rho_{m}, m=1,2, \cdots, M
$$

SSVEP Recognition based on Wavelet CCA. Although the CCA-based methods, like CCA, MCCA, L1-MCCA, etc., have shown quite well performance in SSVEP recognition, all of them pay no attention to reject useless frequency components in EEG signals. Due to the existence of spontaneous EEG and other noises, the collected EEG data usually contain a large number of frequency components which aren't not related to the stimulus frequencies. To overcome this problem, we use wavelet packet decomposition to remove noise frequencies in EEG data. Then, the standard CCA is applied to the refined data to detect its frequency.

Wavelet packet decomposition (WPD) $[11,12]$ is an extension of the discrete wavelet transform (DWT), where the signal is passed through more filters than the DWT. In the DWT, each level is calculated by passing the previous approximation coefficients (low frequency components) through a high and low pass filters. However, both the approximation (low frequency components) and detail (high frequency components) coefficients are decomposed in the WPD, which can provides more information.

After through the WPD, we choose the sub band covering the stimulus frequency, which has the largest energy spectrum, to reconstruct the EEG signal. Next, we can detect its frequency by using the standard CCA.

\section{Experiments and Results}

EEG Data Acquisition. EEG data were collected from three healthy subjects (represented by S1, S2, S3) with normal or corrected-normal vision. During the experiments, all of the three subjects were seated in a comfortable chair $60 \mathrm{~cm}$ from a standard 21 inch LCD monitor $(60 \mathrm{~Hz}$ refresh rate) in a 
shield room. The EEG data were recorded using the Nuamps amplifier (NuAmp, Neuro-scan, Inc) at $250 \mathrm{~Hz}$ sampling rate with high-pass and low-pass filters of 0.1 and $70 \mathrm{~Hz}$ from eight channels $\mathrm{P} 3, \mathrm{Pz}$, P4, P7, P8, O1, Oz and $\mathrm{O} 2$ placed on the standard position of the 10-20 international system. Four white squares on a black screen were flicked as stimuli at four different frequencies: $5.75 \mathrm{~Hz}, 7.75 \mathrm{~Hz}$, $8.75 \mathrm{~Hz}$ and $9.75 \mathrm{~Hz}$. Each subject completed 20 trials under each stimulus frequency. In each trial, the subjects were asked to focus on the four white squares once for $4 \mathrm{~s}$ after each visual cue. At last, we collected 80 experimental data from each subject.

Experimental Evaluation. To confirm effectiveness of our method, we compared it with the standard CCA and MCCA for SSVEP recognition. For MCCA, leave-one-trial-out cross-validation is applied to evaluate the average recognition accuracy. That means the data from 19 trials are used as training data for reference signal extraction while the left-out trial for validation. Fig. 1 presents the average recognition accuracies of the three methods for all the subjects, respectively, with different TWs from 0.5 to $4 \mathrm{~s}$ in different harmonic combinations. The results show that our method yields significantly higher recognition accuracies than the standard CCA for most TWs. Moreover, our method demonstrates quite competitive performance in comparison with MCCA with less computational requirement. We can also learn that, to some extent, more harmonics make better recognition performance. However, too many harmonics cannot always give higher accuracy, since higher order harmonic usually has smaller energy spectrum.

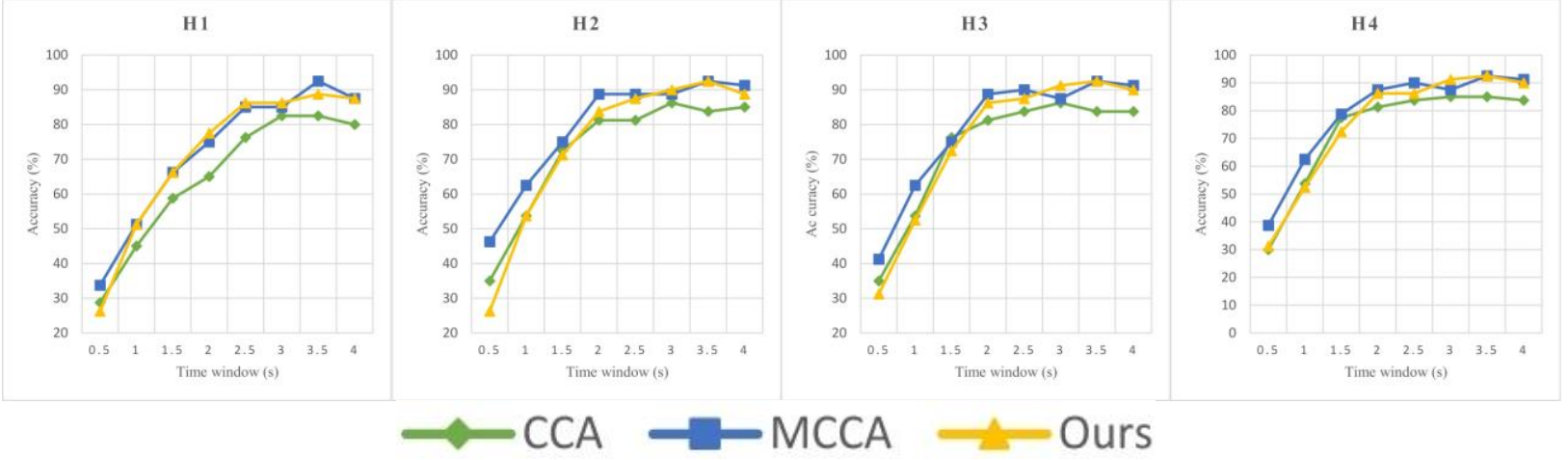

Fig. 1. SSVEP average recognition accuracies of the 3 subjects obtained by the standard CCA, MCCA and our method, with different time window (TW) lengths from 0.5 to $4 \mathrm{~s}$, in different harmonic combinations. H1: fundamental frequency only, H2: fundamental frequency and second harmonic, H3: fundamental frequency, second and third harmonic, H4: fundamental frequency, second, third and forth harmonic.

\section{Conclusions}

In this paper, we introduced wavelet packet decomposition (WPD) which could reject frequency components not related to the stimulus frequency to enhance the CCA-based methods frequency recognition accuracy for SSVEP-based BCIs. In this method, the EEG data were constructed from the sub band wavelet coefficient, which has the largest energy spectrum. After that, multiple linear regression was applied to inspect the correlation between the refined EEG data and sine-cosine reference signals for SSVEP recognition. Experimental results presented our proposed method achieved significantly higher SSVEP recognition accuracies than the standard CCA. Moreover, our method demonstrated quite competitive performance in comparison with MCCA with less computational requirement. In the future, we will exploit other methods to reject useless frequency components and combine them with CCA-based methods, e.g., MCCA, L1MCCA to investigate their effectiveness on accuracy improvement of the SSVEP-based BCIs.

\section{Acknowledgements}

The work was supported by the National Natural Science Foundation of China (Grant Nos. 91420302, 91520201). 


\section{References}

[1] Vallabhaneni A, Wang T, He B. Brain-computer interface[M]//Neural engineering. Springer US, 2005: 85-121.

[2] Martinez P, Bakardjian H, Cichocki A. Fully online multicommand brain-computer interface with visual neurofeedback using SSVEP paradigm[J]. Computational intelligence and neuroscience, 2007, 2007: 13-13.

[3] Jia C, Gao X, Hong B, et al. Frequency and phase mixed coding in SSVEP-based brain--computer interface[J]. IEEE transactions on biomedical engineering, 2011, 58(1): 200-206.

[4] Allison B Z, McFarland D J, Schalk G, et al. Towards an independent brain-computer interface using steady state visual evoked potentials[J]. Clinical neurophysiology, 2008, 119(2): 399-408.

[5] Lin Z, Zhang C, Wu W, et al. Frequency recognition based on canonical correlation analysis for SSVEP-based BCIs[J]. IEEE Transactions on Biomedical Engineering, 2006, 53(12): 2610-2614.

[6] Bin G, Gao X, Yan Z, et al. An online multi-channel SSVEP-based brain-computer interface using a canonical correlation analysis method[J]. Journal of neural engineering, 2009, 6(4): 046002 .

[7] Zhang Y, Zhou G, Zhao Q, et al. Multiway canonical correlation analysis for frequency components recognition in SSVEP-based BCIs[C]//International Conference on Neural Information Processing. Springer Berlin Heidelberg, 2011: 287-295.

[8] Zhang Y, Zhou G, Jin J, et al. L1-regularized multiway canonical correlation analysis for SSVEP-based BCI[J]. IEEE Transactions on Neural Systems and Rehabilitation Engineering, 2013, 21(6): 887-896.

[9] Hotelling H. Relations between two sets of variates[J]. Biometrika, 1936, 28(3/4): 321-377.

[10]Wei Q, Xiao M, Lu Z. A comparative study of canonical correlation analysis and power spectral density analysis for SSVEP detection[C]//Intelligent Human-Machine Systems and Cybernetics (IHMSC), 2011 International Conference on. IEEE, 2011, 2: 7-10.

[11] Ting W, Guo-zheng Y, Bang-hua Y, et al. EEG feature extraction based on wavelet packet decomposition for brain computer interface[J]. Measurement, 2008, 41(6): 618-625.

[12] Zhang Y, Liu B, Ji X, et al. Classification of EEG Signals Based on Autoregressive Model and Wavelet Packet Decomposition[J]. Neural Processing Letters, 2016: 1-14. 